\title{
Frontal Lobe Volume, Function, and $\beta$-Amyloid Pathology in a Canine Model of Aging
}

\author{
P. Dwight Tapp, ${ }^{1,4}$ Christina T. Siwak, ${ }^{2,5}$ Fu Qiang Gao, ${ }^{3}$ Jr-Yuan Chiou, ${ }^{4}$ Sandra E. Black, ${ }^{1,2,3}$ Elizabeth Head, ${ }^{5}$ \\ Bruce A. Muggenburg, ${ }^{6}$ Carl W. Cotman, ${ }^{5}$ Norton W. Milgram, ${ }^{1,2}$ and Min-Ying Su ${ }^{4}$ \\ ${ }^{1}$ Department of Psychology, ${ }^{2}$ Institute of Medical Science, ${ }^{3}$ Sunnybrook and Women's College Health Sciences Centre, University of Toronto, Scarborough, \\ Ontario M1C 1A4, Canada, ${ }^{4}$ Center for Functional Onco-Imaging, and ${ }^{5}$ Institute for Brain Aging and Dementia, University of California, Irvine, California \\ 92697, and ${ }^{\circ}$ Lovelace Respiratory Research Institute, Albuquerque, New Mexico 87115
}

Application of magnetic resonance imaging (MRI) techniques reveals that human brain aging varies across cortical regions. One area particularly sensitive to normal aging is the frontal lobes. In vitro neuropathological studies and behavioral measures in a canine model of aging previously suggested that the frontal lobes of the dog might be sensitive to aging. In the present study, MRI scans were acquired to compare age-related changes in frontal lobe volume with changes in executive functions and $\beta$-amyloid pathology in the frontal cortex of beagle dogs aged 3 months to 15 years. Decreases in total brain volume appeared only in senior dogs (aged 12 years and older), whereas frontal lobe atrophy developed earlier, appearing in the old dogs (aged 8-11 years). Hippocampal volume also declined with age, but not occipital lobe volume past maturity. Reduced frontal lobe volume correlated with impaired performance on measures of executive function, including inhibitory control and complex working memory, and with increased $\beta$-amyloid accumulation in the frontal cortex. Age-related hippocampal atrophy also correlated with complex working memory but not inhibitory control, whereas occipital lobe volume did not correlate with any cognitive measure. These findings are consistent with the frontal lobe theory of aging in humans, which suggests that the frontal lobes and functions subserved by this region are compromised early in aging.

Key words: brain volume; frontal lobe; aging; beagle; MRI; amyloid

\section{Introduction}

Aging is associated with variable changes in brain volume, morphology, and cognitive function. One brain region that may be particularly vulnerable to aging is the frontal cortex. In humans, rates of cortical decline in the frontal lobes are typically greater than rates for the entire brain, hippocampal complex, amygdala, parietal, occipital, and insular cortices (Coffey et al., 1992; Murphy et al., 1996; Raz, 1996; Salat et al., 1999, 2001; Resnick et al., 2000; Jernigan et al., 2001). Decline is also larger in the dorsolateral and orbitofrontal cortex compared with the frontal pole, anterior cingulate, or precentral gyrus (Jernigan et al., 1991; Raz et al., 1993, 1997; Tisserand et al., 2002).

Patterns of $\beta$-amyloid $(\mathrm{A} \beta)$ deposition in healthy aged humans (Braak and Braak, 1997; Bussière et al., 2002) and nonhuman primates (Struble et al., 1985; Heilbroner and Kemper,

Received April 8, 2004; revised July 19, 2004; accepted July 22, 2004.

This work was supported by grants from the National Institute on Aging (AG12694 and AG17066) and the United States Army Medical Research and Material Command under Contract DAMD17-98-1-8622. We thank Joseph Araujo, Lori-Ann Christie, Christa Studzinski, Jimena Estrada, Joan Chan, Candace lkeda-Douglas, Cleo Leung, Winnie Lau, Sarah Skoggard, Dr. Jean Douglas, Dr. Jane Bridgeman, Dr. Wendy Williams, Heather Murphey, and the staff at Lovelace Respiratory Research Institute for technical expertise and support in acquiring magnetic resonance imaging data for this study.

The views, opinions, and/or findings contained in this report are those of the authors and should not be construed as an official Department of the Army position, policy, or decision unless so designated by other documentation.

Correspondence should be addressed to Dr. P. Dwight Tapp, Center for Functional Onco-Imaging, University of California, 164 Irvine Hall, Irvine, CA 92697-5020. E-mail: ptapp@uci.edu.

DOI:10.1523/JNEUROSCI.1339-04.2004

Copyright $\odot 2004$ Society for Neuroscience $\quad 0270-6474 / 04 / 248205-09 \$ 15.00 / 0$
1990) also suggest that frontal lobe integrity is compromised early in aging. In aging human brains, $A \beta$ deposition occurs early in the prefrontal cortex (Braak and Braak, 1997; Bussière et al., 2002). In vitro studies in beagle dogs report similar variability in $\mathrm{A} \beta$ accumulation with the earliest and most consistent deposition occurring in the prefrontal cortex at $\sim 8$ years of age (Yoshino et al., 1996; Hou et al., 1997; Satou et al., 1997; Head et al., 2000). In contrast, $A \beta$ deposition in the entorhinal, parietal, and occipital brain regions does not appear consistently until after 12 years of age in the dog (Head et al., 2000).

Consistent with the frontal lobe theory of aging, complex cognitive processes, such as categorical abstraction, monitoring, manipulation, shifting of set, and inhibitory control, decrease after damage to the frontal lobes or in parallel with age-related decreases in frontal lobe volume (FLV) in humans (Milner, 1982; Raz et al., 1998b; Stuss et al., 2001; Gunning-Dixon and Raz, 2003), monkeys (Petrides, 1991, 1995; Dias et al., 1996), and rats (Iversen and Mishkin, 1970; Birrell and Brown, 2000; Delatour and Gisquet-Verrier, 2001). We previously reported impaired executive functions in aged beagle dogs on measures of inhibitory control (Tapp et al., 2003a), maintenance and manipulation (Tapp et al., 2003b), and concept abstraction (Tapp et al., 2004).

The present study examined the effect of age on frontal lobe volume in young and old dogs using in vivo magnetic resonance imaging (MRI). In a previous MRI study, decreased total brain volume (TBV) and increased ventricular volume were observed in aging beagle dogs (Su et al., 1998). Although these findings are 
similar to ventricular and cortical changes in human MRI studies (Condon et al., 1988; Gur et al., 1991; Sullivan et al., 2002), regional differences in brain volume as a function of age in the dog were not examined. The present study is the first to apply MRI and image processing techniques to selectively examine in vivo frontal lobe aging in the beagle dog. Changes in frontal lobe volume were subsequently compared with $\mathrm{A} \beta$ deposition and executive functions to develop a more comprehensive analysis of frontal lobe aging in the canine model.

\section{Materials and Methods \\ Subjects}

The study was performed with beagle dogs housed at the University of Toronto (Ontario, Canada) and the Lovelace Respiratory Research Institute (LRRI; Albuquerque, NM). Animals were housed singly or in pairs in vivarium cages at University of Toronto or in indoor/outdoor kennel runs at LRRI. Fresh water was provided ad libitum, and each subject received $300 \mathrm{gm}$ of food (Hill's Pet Nutrition; Canine Maintenance, Topeka, KS) daily. Animals were inspected daily by trained veterinary and behavioral technicians and clinical neurological examinations were performed annually. All of the dogs were in good health before the study, and procedures were conducted in accordance with local and federal animal care policies.

\section{Imaging procedures}

Magnetic resonance imaging procedures were conducted using a GE-LX 1.5 T mobile MRI scanner. MRI scans were collected for 66 beagles (32 males, 34 females) aged 3 months to 15 years. During imaging procedures, each dog was sedated by a subcutaneous injection of acepromazine ( $2 \mathrm{mg}$ ), and anesthesia was then induced by inhalation of $5 \%$ isoflurane and maintained using $2 \%$ isoflurane-oxygen gas mixture. Respiration was maintained with a Bellows respirator, and heart rate was monitored through an esophageal balloon. Subjects were placed into the magnet bore headfirst in a prone position, and a quadrature knee radiofrequency coil was secured over the head of the animal. Imaging parameters included two excitations (number of excitations, 2.0), a $256 \times 256$ matrix, and a $12 \mathrm{~cm}$ field of view. $\mathrm{T}_{1}$-weighted brain images were acquired in the coronal plane using a spoiled gradient (spoiled gradient-recalled acquisition in a steady state) pulse sequence (repetition time, $40 \mathrm{msec}$; echo time, $9.0 \mathrm{msec}$; flip angle, $40^{\circ}$; slice thickness, $1.2-1.4 \mathrm{~mm}$; pixel size, 0.47). Sixty images were acquired for each dog during a 25 min scan period. After completing the scan, animals were recovered in isolation cages under observation of the facility veterinarian.

\section{Reformatting and alignment of $M R$ images}

Variations in head tilt, pitch, and rotation were corrected off-line using the proprietary software Analyze (version 4.0; Mayo Clinic, Rochester, $\mathrm{MN}$ ), with standard neuroanatomical landmarks (Talairach and Tournoux, 1988), to bring images into a unified coordinate system in all three orthogonal planes. Deviations in head rotation were corrected in the axial plane by adjusting the ocular orbits perpendicular to the interhemispheric fissure. Variations in pitch were corrected by aligning images in the axial and sagittal planes along the anterior-posterior commissures. Head tilt was corrected in the axial plane by aligning images with the orbital and auditory canals. All of the region-of-interest (ROI) analyses were performed on reformatted images (Fig. 1).

\section{Computer analysis of MRI scans}

Manual planimetry techniques were used to trace the following ROIs: the frontal cortex (total, left and right), left and right ventricles within the frontal cortex [i.e., frontoventricular volume (FVV)], total brain volume (including separate ROIs for left and right cerebral hemispheres), total intracranial volume (TIV), hippocampal volume (HCV), and occipital lobe volume (OCV).

The frontal cortex was defined in the coronal plane beginning with the olfactory bulbs and continued rostrocaudally through contiguous slices to the anterior cruciate sulcus. The cruciate sulcus was demarcated from a three-dimensional surface rendering of the entire brain achieved by automatic erosion of nonparenchymal areas in Analyze. Total frontal lobe volume was acquired by tracing the left and right frontal lobes separately on contiguous slices throughout the entire canine frontal cortex. When visible, left and right lateral ventricles in the frontal lobes were also traced as separate objects.

TBV and TIV were defined in the transaxial plane beginning with the first slice at the level of the ventral pons. TBV and TIV measures were acquired from every third slice ventrodorsally to the superior-most slice in the dorsal plane of the cerebral cortex.

The hippocampus was traced from the most anterior to the most posterior section where the structure was visible. Occipital lobes were demarcated using the superior colliculi as the anterior landmark to the most posterior section displaying the cortex.

TBV was calculated as a proportion of the TIV. FLV, FVV, HCV, and OCV were calculated as a ratio of TBV. This resulted in the following brain volume variables for statistical analysis: percentage of total brain volume $[(\mathrm{TBV} / \mathrm{TIV}) \times 100]$, percentage of total frontal volume [(FLV/ $\mathrm{TBV}) \times 100$ ], percentage of left frontal lobe volume [(left FLV/left hemisphere $) \times 100]$, percentage of right frontal lobe [(right FLV/right hemisphere $) \times 100]$, percentage of frontoventricular volume $[($ FVV/FLV $) \times$ $100]$, percentage of hippocampal volume $[(\mathrm{HCV} / \mathrm{TBV}) \times 100]$, and percentage of occipital volume $[(\mathrm{OCV} / \mathrm{TBV}) \times 100]$.

\section{Reliability of regional volumetric measurements}

All of the ROIs were performed by the same person (P.D.T.). These measures were repeated by a second rater (C.T.S.) on 30 randomly selected images to obtain intraclass correlation (ICC) measures for all of the ROIs. ICC reliability estimates for all of the ROIs exceeded 0.958 .

\section{Cognitive tasks}

Cognitive measures were performed within 3-12 months of the MRI scans and were conducted in a $0.609 \times 1.15 \times 1.08$ m wooden canineadaptation of the Wisconsin General Test Apparatus (Milgram et al., 1994). The battery of cognitive tests included an initial size and reversal discrimination task (Head et al., 1998; Tapp et al., 2003a), a complex working memory task (Tapp et al., 2003b), and a set learning and size concept task (Tapp et al., 2004).

Size discrimination and reversal. Inhibitory control was measured using the size discrimination and reversal task. On the size discrimination task, subjects were presented with two identical red wooden blocks differing only in height. Responses to only one object (i.e., large or small block) resulted in a food reward. Each subject received a total of 10 trials per day, and the location of the large and small objects was randomized between trials. One-half of the animals on the size discrimination task were rewarded for choosing the large object, and the remaining one-half were rewarded for choosing the small object. After completing the size discrimination task, all of the animals were tested on size discrimination reversal (SDR) procedures. Test procedures for this task were identical with the size discrimination task except that the reward contingencies were reversed. Thus, animals previously rewarded for choosing the large block were now rewarded for selecting the small block, and animals rewarded for choosing the small block were rewarded for choosing the large block. A total of 46 animals (22 males, 24 females), aged 3-14 years, were tested on the size discrimination and SDR tasks.

Spatial list learning. The spatial list learning (SLL) task measured complex working memory processes, including maintenance and manipulation of information. Two subtests, SLL and modified SLL (mSLL), were used. Each subtest consisted of three phases per trial. On the SLL task, subjects were presented with a single red coffee jar lid in one of three spatial locations on phase 1 of each trial. After a fixed delay $(5,10,20$, or $50 \mathrm{sec}$ ), a second identical coffee jar lid was presented on phase 2 of the same trial. This second stimulus was presented simultaneously with the first object, in one of the two remaining spatial locations. Only responses to the object in the new spatial location were rewarded. After a second delay period $(5,10$, or $20 \mathrm{sec})$, a third identical coffee jar lid was presented simultaneously with the first two objects, located in the last remaining spatial location. Only responses to this object were rewarded. Subjects were tested on the SLL subtest for a maximum of $50 \mathrm{~d}$. Testing on the mSLL subtest began the first day after the SLL task. Testing procedures on the mSLL were identical with those used on the SLL task, with the fol- 


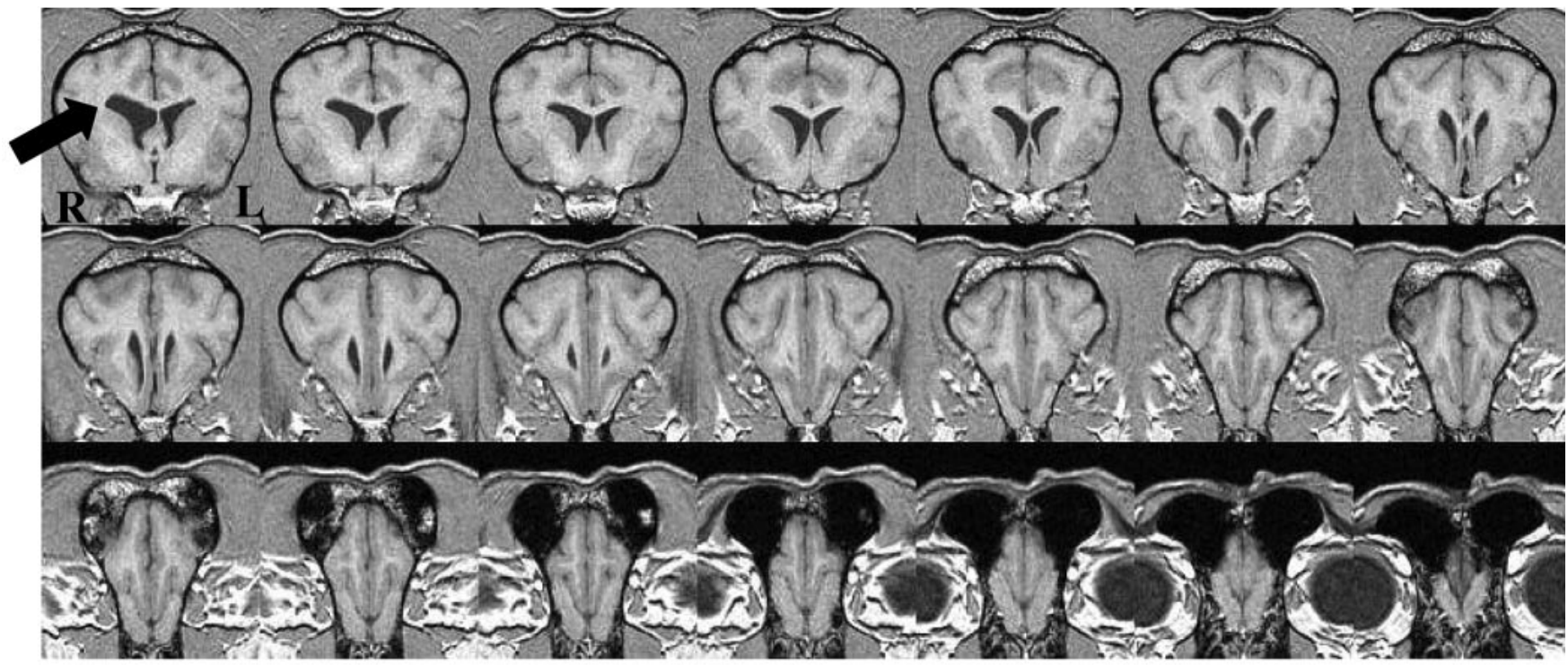

A

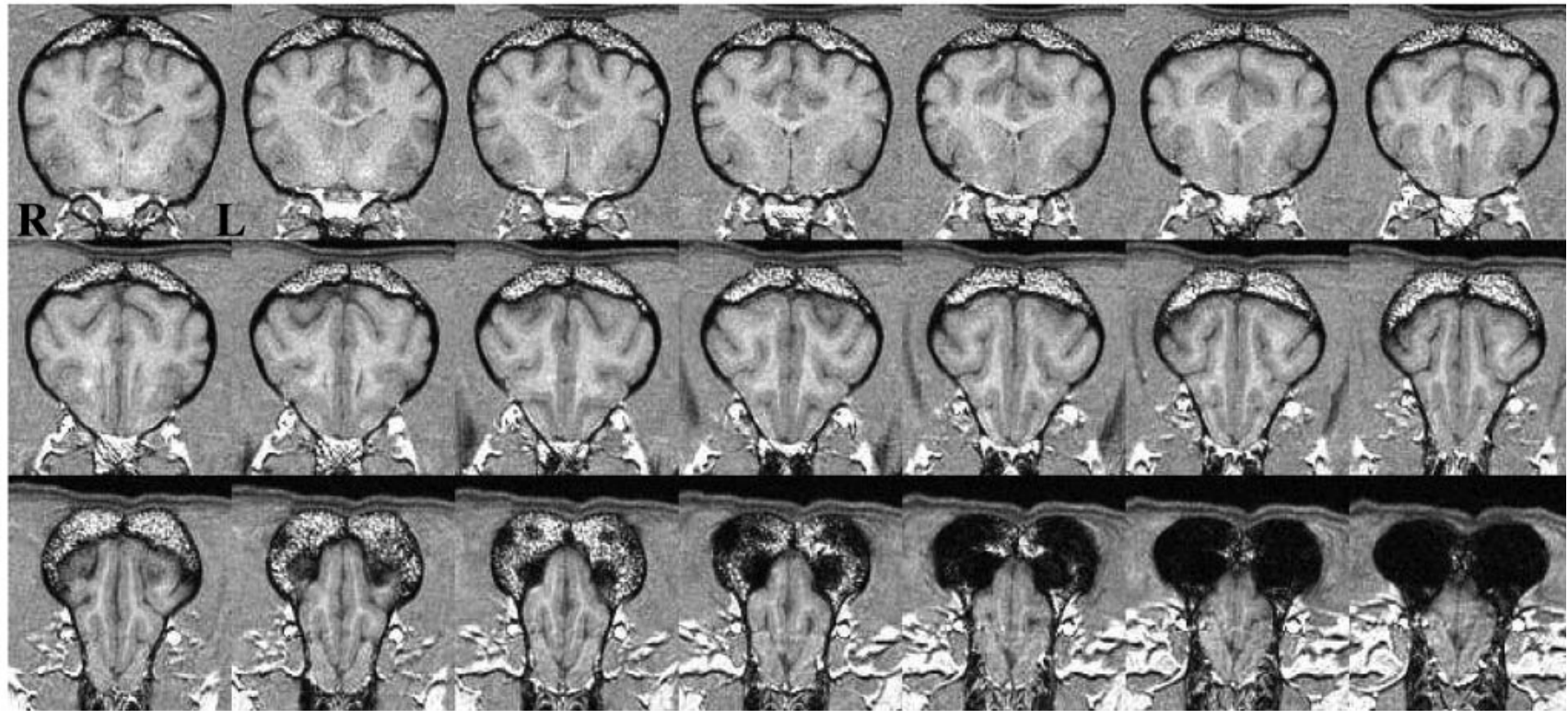

B

Figure 1. Contiguous coronal slices from an MRl in a senior $(A)$ and young $(B)$ dog brain through the frontal lobes displayed in a caudal-rostral direction [left $(\mathrm{L})$ to right $(\mathrm{R})]$ from the cruciate sulcus to the anterior pole of the olfactory bulbs. Separate ROIs were calculated for left and right frontal hemispheres and ventricular volumes (arrow) for each subject. Identical planimetry procedures were used to calculate left, right, and total brain volumes in the transaxial plane for each dog.

lowing exception: on phase 2 of each trial, the second coffee jar lid was presented in the absence of the first coffee jar lid (i.e., only one coffee jar lid was present during phases 1 and 2 of each trial). Twelve animals (nine males, three females), aged $4-15$ years, completed the SLL task, and 10 of those animals (eight males, two females) completed the mSLL task.

Size learning set discrimination and concept learning. The size discrimination protocol described above was modified to assess rule induction and concept learning in the dogs. Measurement of these executive functions involved three related tasks: a two-choice size discrimination (2CSD) learning-set task, a three-choice size discrimination (3CSD) task, and a three-choice size concept (3CSC) task. The 2CSD task consisted of six different subtests, each involving a size discrimination between two red blocks. Subtest 1 used the same large and small blocks from the initial size discrimination task. After reaching criterion on subtest 1 , subjects were tested on the remaining five subtests using a fixed order of testing. On subtest 2, animals were presented with a discrimination problem between a two- and three-block stimulus; on subtest 3, a three- and four-block stimulus; on subtest 4 , a one- and four-block stimulus; on subtest 5 , a two- and four-block stimulus; and on subtest 6 , a one- and three-block stimulus. Twenty-two animals ( 9 males, 13 females), aged 4-15 years, were tested on the 2CSD task.

Testing on the 3CSD began the first day after the last 2CSD subtest and included 19 of the 22 animals ( 7 males, 12 females) from the 2CSD task. Stimuli 1, 2, and 4 from the 2CSD subtests were used for this task. Sub- 
jects were rewarded for selecting the size of object that was rewarded during the 2CSD task. Spatial locations of all three objects were randomized across each daily session of 12 trials.

The final cognitive measure was the $3 \mathrm{CSC}$ task, which consisted of two subtests: the 3CSCballs and 3CSC-bottles tasks. On the 3CSCballs task, dogs were presented with three toy soccer balls varying in size (small, medium, and large). Only responses to objects conceptually similar in size to those used on the 3CSD task were rewarded. Thus, animals rewarded for selecting the smallest block on the 3CSD task were rewarded for choosing the smallest toy soccer ball on the 3CSC task. Object locations were randomized between three spatial locations across 12 trials per session. After completing the 3CSC-balls task, subjects were tested on the 3CSC-bottles task in which stimuli consisted of three identical shampoo bottles varying in height.

\section{A $\beta$ quantification}

Several months after the MRI scans, 23 dogs (12 males, 11 females; aged 2-15 years) were killed, and their brains were removed according to a standard protocol. The tissue was transferred into PBS, pH 7.4 , with $0.02 \%$ sodium azide and stored at $4^{\circ} \mathrm{C}$. $\mathrm{A} \beta$ was detected using a rabbit polyclonal antibody $\mathrm{A} \beta_{42}$, raised against a synthetic $\beta$-amyloid peptide (Biosource International, Camarillo, CA) that detects amino acids $1-42$ of the $\mathrm{A} \beta$ peptide. Forty-micrometerthick vibratome sections were taken from the prefrontal cortex according to a canine brain atlas (Kreiner, 1966). Sections were pretreated for $4 \mathrm{~min}$ with $90 \%$ formic acid before overnight incubation at room temperature in $\mathrm{A} \beta_{42}(1: 5000)$ in Tris-saline with $2.0 \%$ BSA and $0.1 \%$ Triton X-100. Bound antibody was detected using a biotinylated anti-rabbit $\mathrm{ABC}$ peroxidase kit from Vector Laboratories (Burlingame, $C A$ ). $A \beta_{42}$ was visualized using a DAB substrate kit from Vector Laboratories.

The area occupied by $\beta$-amyloid was quantified using gray-scale thresholding procedures (Cummings and Cotman, 1995; Cummings et al., 1996). Ten semirandom fields, five in the superficial and five in the deep layers, of the frontal cortex were centered and digitized at $20 \times$. Staining was captured using a $2.5 \times$ photo eyepiece, a Sony (Tokyo, Japan) high-resolution CCD video camera (XC-77), and the built-in video capture capabilities of a Macintosh 8100/80AV. Using NIH Image 1.59, grayscale thresholding at a cutoff level of 110 was selected to separate positive staining from background and to calculate the percentage of area occupied by $\beta$-amyloid immunoreactivity. Units of $\mathrm{A} \beta$ represent the average percentage of 10 individual $525 \times 410 \mu \mathrm{m}$ fields per region occupied by positive $\mathrm{A} \beta$ staining and are arbitrary, because the magnification chosen directly affects the final value.

\section{Statistics}

Regression techniques were used to examine the relationship between frontal lobe volume with age, cognitive function, and $A \beta$ loads in the frontal cortex, as well as HCV and OCV with age and cognition. Effects of age and sex on corrected TBV, FLV, FVV, HCV, and OCV were also examined using a general linear multivariate ANOVA. Post hoc analyses were performed using the Tukey's honestly significant difference procedure. All of the analyses were performed using SPSS, version 11.5. Differences were considered significant at $p<0.05$ (two-tailed) for all of the tests.

\section{Results}

\section{Brain volumes}

Changes in TBV, FLV, FVV, and HCV as a function of chronological age are shown in Figure 2. Increasing age was associated

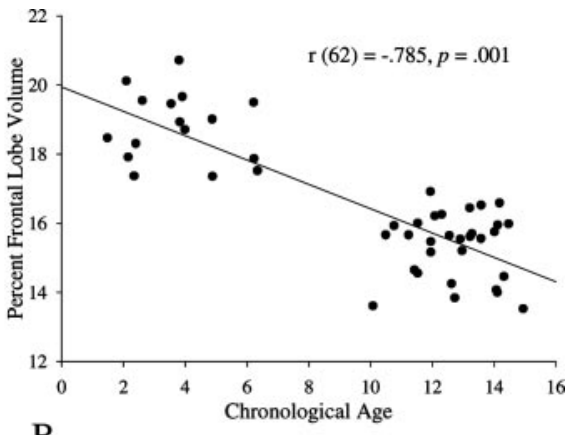

B

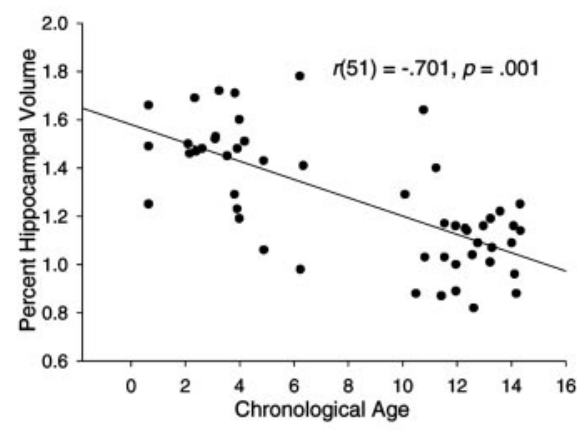

D

with smaller TBV $\left(r_{(30)}=-0.846 ; p=0.0001\right)$, FLV $\left(r_{(62)}=\right.$ $-0.785 ; p=0.0001)$, and $\operatorname{HCV}\left(r_{(51)}=-0.701 ; p=0.001\right)$, and larger FVV $\left(r_{(66)}=0.511 ; p=0.0001\right)$. OCV did not vary with chronological age $\left(r_{(55)}=-0.130 ; p=0.362\right)$. To obtain a qualitative description of age on brain volume, male and female subjects were separated into five age groups based on previous criteria (Tapp et al., 2003a): puppies ( 3 months of age), young dogs (6 months to 3.9 years of age), middle-aged dogs (4-7.9 years of age), old dogs ( $8-11.4$ years of age), and senior dogs ( 11.5 years and older). A significant main effect of age was observed for TBV $\left(F_{(4,21)}=36.25 ; p=0.0001\right), \operatorname{FLV}\left(F_{(4,56)}=14.80 ; p=0.0001\right)$, $\operatorname{FVV}\left(F_{(4,56)}=6.07 ; p=0.0001\right), \operatorname{HCV}\left(F_{(4,40)}=14.51 ; p=\right.$ $0.0001)$, and $\operatorname{OCV}\left(F_{(4,40)}=2.97 ; p=0.031\right)$.

TBV was much smaller in senior dogs (Fig. 3) compared with all of the other groups $(p<0.001)$. FLV (Fig. 4) in senior dogs was also smaller compared with middle-aged $(p=0.003)$ and young dogs $(p=0.0001)$, and in old dogs compared with young dogs $(p=0.004)$. FVV (Fig. 5) was also much larger in senior dogs compared with old $(p=0.031)$ and young $(p=0.002)$ dogs, and puppies $(p=0.005)$, but did not differ from middle-aged $(p=$ 0.811 ). This was likely attributable to one animal in the middle-aged group whose percentage of FVV was 1.89 SDs greater than average frontoventricular volume for dogs in that group. Removing this animal from the analysis resulted in a significant difference in FVV between senior and middle-aged dogs $(p=0.039)$.

Corrected HCV (Fig. 6) was smaller in puppies compared with young $(p<0.001)$, middle-aged $(p=0.002)$, and old dogs $(p=0.033)$, and in senior dogs, HCV was smaller compared with middle-aged $(p=0.020)$ and young dogs $(p<0.001)$. HCV in old and senior dogs, however, did not differ significantly $(p>$ $0.05)$. Corrected OCV was larger only in the puppies compared 


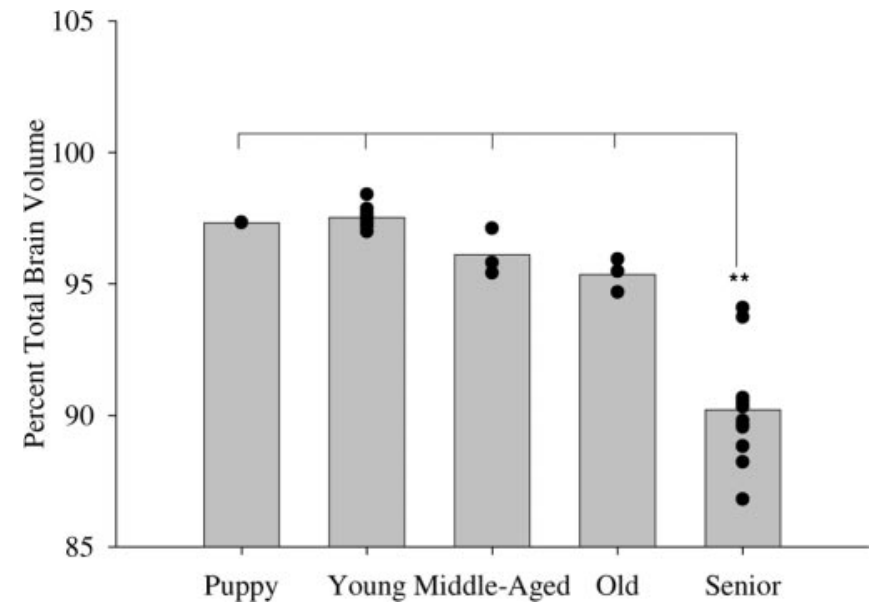

Figure 3. Mean total brain volume as a proportion of total intracranial volume in puppies and young, middle-aged, old, and senior dogs. Individual values for animals within each group are presented, and significantly different groups are indicated by asterisks $\left({ }^{* *} p<0.01\right)$.

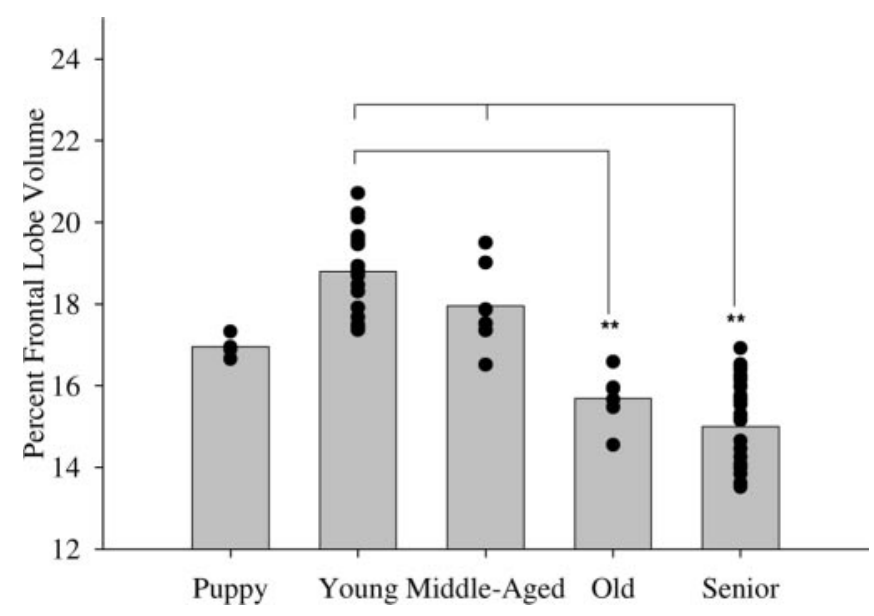

Figure 4. Mean frontal lobe volume as a proportion of total brain volume in puppies and young, middle-aged, old, and senior dogs. Individual values for animals within each group are presented, and significantly different groups are indicated by asterisks $\left({ }^{* *} p<0.01\right)$.

with young $(p=0.013)$, old $(p=0.022)$, and senior dogs $(p=$ 0.032 ).

In contrast to the main effect of age, there was no significant main effect of sex, or interaction between sex and age on any of the corrected brain measures $(p>0.05)$.

\section{Brain-behavior relationships}

A relationship between FLV and complex cognitive processes was observed on several indices of executive function. On the initial size and reversal discrimination tasks (Fig. 7), errors increased as FLV decreased $\left(r_{(46)}=-0.285, p=0.037 ; r_{(46)}=-0.325, p=\right.$ 0.020; respectively). On the mSLL task (Fig. 8), larger FLVs were associated with fewer total errors $\left(r_{(10)}=-0.516 ; p=0.050\right)$ and higher maximal memory scores $\left(r_{(10)}=0.677 ; p=0.011\right)$. Percentage of correct scores on the 2CSD set-learning task generally increased with FLV, but the relationship between FLV and percentage of correct scores was only significant on the last subtest $\left(r_{(22)}=0.592 ; p=0.002\right)$. A similar trend was also observed on the 3CSC-balls and 3CSC-bottles tasks with percentage of correct scores on both tasks increasing with FLV (Fig. 9), but the relationship was not statistically significant $(p>0.05)$.

Smaller HCVs were associated with increased errors on

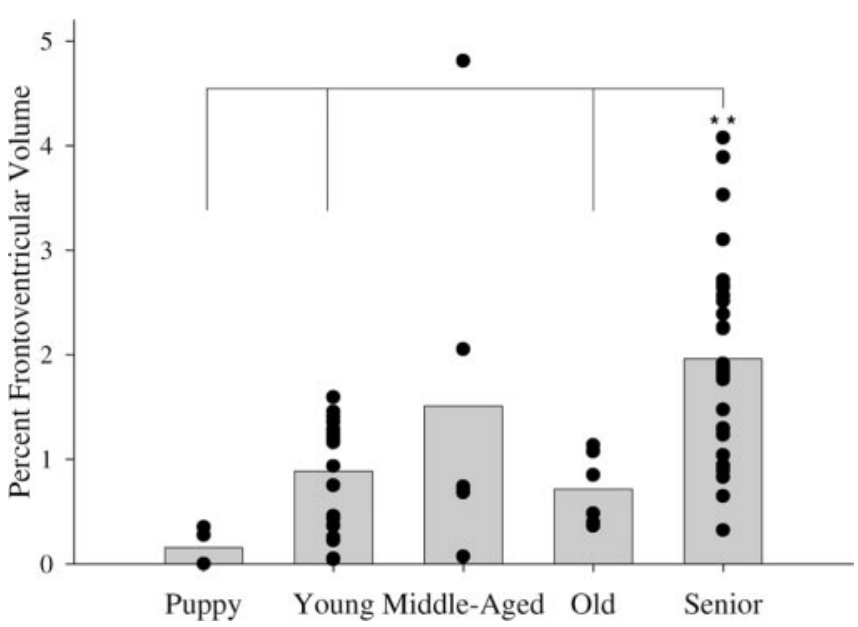

Figure 5. Mean frontoventricular volume expressed as a proportion of total frontal lobe volume in puppies and young, middle-aged, old, and senior dogs. Individual values for animals within each group are presented, and significantly different groups are indicated by asterisks $\left({ }^{* *} p<0.01\right)$.

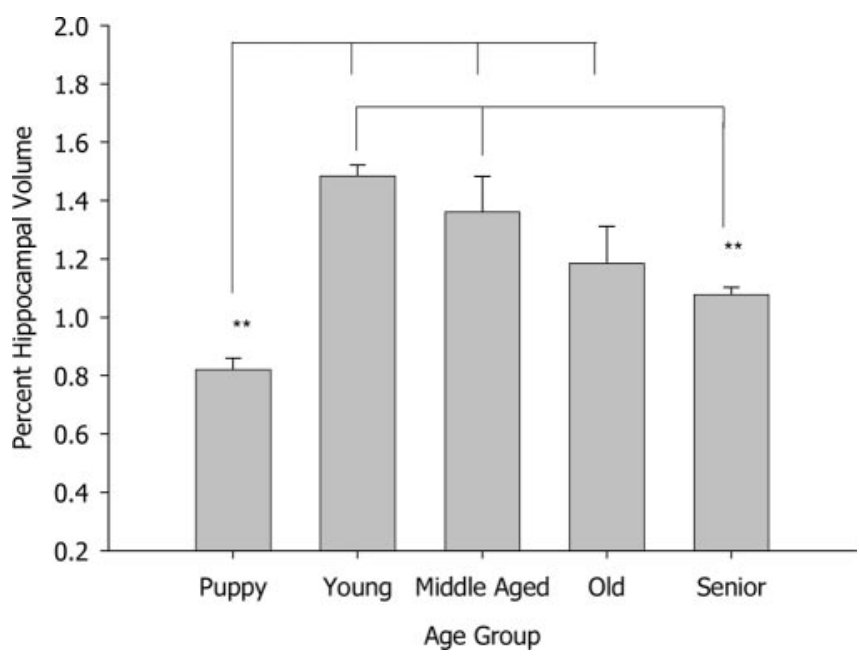

Figure 6. Mean hippocampal volume expressed as a proportion of total brain volume in puppies and young, middle-aged, old, and senior dogs. Individual values for animals within each group are presented and significantly different groups are indicated by asterisks $\left({ }^{* *} p<\right.$ $0.01)$.

subtest 3 of the 2CSD task $\left(r_{(35)}=-0.572 ; p=0.008\right)$ and the mSLL task $\left(r_{(11)}=-0.771 ; p=0.003\right)$, whereas larger HCVs were associated with increased percentage of correct scores on subtest 1 of the 3CSC task $\left(r_{(18)}=0.464 ; p=0.036\right)$ and higher maximal memory scores on the mSLL task $\left(r_{(10)}=0.767 ; p=\right.$ $0.003)$. OCV was not related to performance on any cognitive measure $(p>0.05)$.

\section{Frontal lobe volumes and $\boldsymbol{\beta}$-amyloid}

Regression analyses also indicated a strong relationship between volume of the frontal cortex and $\mathrm{A} \beta$ loads in the superficial and deep layers of the frontal cortex (Table 1). Smaller FLVs were associated with larger average amyloid loads and amyloid loads in the superficial layer of the frontal cortex $(p<0.01)$. A similar trend was observed between FLV and levels of amyloid in deeper layers of the frontal cortex, but the correlation did not achieve statistical significance $(p>0.05)$. 


\section{Discussion}

The present study used in vivo MRI and manual planimetry procedures to examine age-related changes in the FLV of beagle dogs. Consistent with a previous study ( $\mathrm{Su}$ et al., 1998), TBV decreased with age. From 3 months to 11 years of age, TBV remained stable in the dog. Decreases in TBV, however, were observed in dogs aged 12 years and older (i.e., senior dogs). Quantification of the anterior horn of the lateral ventricles indicated that frontoventriculomegaly was almost two times greater in senior dogs compared with young, old, and middle-aged dogs, and 13 times larger compared with puppies. This area of the lateral ventricle was selected as a region of interest, because enlargement of this area was unique to older dogs. Increased ventricular volume is a hallmark of aging in human MRI studies (Condon et al., 1988; Sullivan et al., 2002). Two previous in vivo studies in aging dogs reported increased total ventricular volume with age (Vite et al., 1997; Su et al., 1998), a finding later confirmed in vitro (González-Soriano et al., 2001). Dilation of the anterior portion of the lateral ventricle may represent a hallmark of advanced age in the dog, but it is unclear from the present study whether the degree of dilation is any greater than the total dilation of ventricles in old dogs.

Decreased FLV with age was also observed in the dog, consistent with human MRI studies of frontal lobe aging (Coffey et al., 1992; Raz et al., 1993, 1997; Murphy et al., 1996; Salat et al., 1999, 2001; Jernigan et al., 2001; Tisserand et al., 2002). Age-related changes in FLV in the present study were nonlinear. In puppies, the ratio of FLV to TBV was smaller compared with all of the other dogs, suggesting that, at 3 months of age, the frontal lobes in these animals had not reached full maturity. These findings are comparable with the in vitro results reported by Fox (1971), indicating that the beagle brain does not reach full maturation until 4-6 months of age when myelination of the frontal region is complete. Beyond 6 months of age, frontal lobe volume remained stable until $\sim 7$ years of age. After 8 years of age, a significant decrease in FLV was observed. This age-related decrease in FLV preceded decreases in TBV, which were greatest in the senior dogs aged 12 and older, and temporally corresponds to $\mathrm{A} \beta$ deposition in the frontal lobes of the dog (Head et al., 2000). Performance on tests designed to measure executive functions (Tapp et al., 2003a,b, 2004), comparable with those used in human studies, declined with decreasing FLV in aging dogs.

The occipital region undergoes the most rapid development postnatally in beagles and is fully matured before the frontal cortex (Fox, 1971). The larger percentage of OCV observed in the puppies likely reflects this development, because no ageassociated decrease in volume was observed in the adult dogs. There was no correlation between OCV and cognitive tasks used in the current study. This suggests that the frontal lobes may be a cortical area particularly sensitive to aging in adult dogs.

Hippocampal volume also showed a decrease with age past maturity and similar correlations with cognitive performance as the frontal lobe. This suggests that the hippocampus is also sensitive to aging and may be involved in the cognitive processes engaged by the tasks used. This is not surprising given the importance of the hippocampus in learning and executive functions (Winocur and Moscovitch, 1990; Lombardi et al., 1999). The

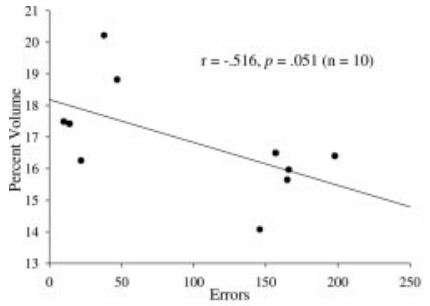

A

Figure 8. Relationships between frontal lobe volume and total errors to criterion $(A)$ and maximal memory score $(B)$ on the mSLL task in 10 beagle dogs.

smaller HCV in the puppies indicates the hippocampus has not completely matured similar to the frontal lobes.

The present study revealed several important and novel findings concerning the frontal lobes in aging dogs. First, changes in FLV follow a nonlinear pattern, increasing until middle age and declining thereafter. Second, age-related frontal lobe atrophy occurs early in aging. In the present study, declining FLV was observed at 10 years of age but may occur even earlier in the dog, because there were no animals between 7 and 9 years of age in this study. Third, shrinkage of the frontal lobes in the dog is concurrent with neuropathological changes in the same brain region. Fourth, cortical atrophy of the frontal lobes in aging dogs is associated with decreased performance on measures sensitive to frontal lobe functioning but also involve other brain regions such as hippocampus.

\section{Mechanisms of age-related frontal lobe atrophy in the dog}

The results from the MRI volumetric analysis provide the first in vivo evidence of decreasing FLV in the beagle dog beginning at $\sim 10$ years of age. Additionally, these changes correlate with increased $\mathrm{A} \beta$ deposition in the frontal lobes and impaired executive functions.

Our previous work shows that $\mathrm{A} \beta$ deposition is age dependent and correlates with cognition (Head et al., 1998). Furthermore, $\mathrm{A} \beta$ is toxic to neurons (Pike et al., 1993) and may be one mechanism mediating cell loss and thus atrophy in the prefrontal cortex. Our observation of a link between frontal atrophy and more extensive $\mathrm{A} \beta$ deposition provides additional support for this hypothesis. Other structural changes in neurons are also plausible. For example, human MRI studies suggest that age-related decreases in FLV represent gray matter loss (Murphy et al., 1996; Raz et al., 1997; Ge et al., 2002) resulting from decreased glycosphingolipids (Kracun et al., 1991, 1992), dendritic arbor (Coleman and Flood, 1987; Terry et al., 1987), or neuronal 


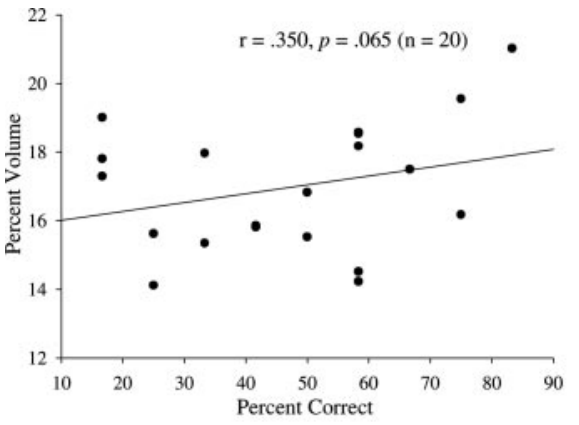

A

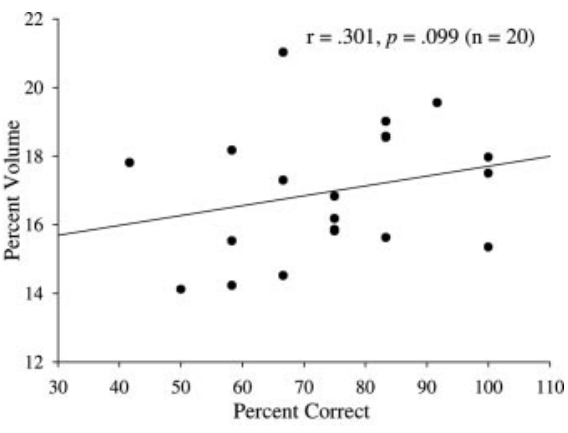

B

Figure 9. Relationship between percentage of frontal lobe volume and percentage of correct scores on the $3 \operatorname{CSC}-$ balls $(A)$ and 3CSC-bottles $(B)$ tasks in 19 beagle dogs.

Table 1. Relationship between brain volume and amyloid loads in the frontal cortex of the beagle dog

\begin{tabular}{lll}
\hline & $r$ & $p$ Value \\
\hline Superficial layer & -0.504 & 0.007 \\
Deep layer & -0.319 & 0.069 \\
Total average & -0.384 & 0.035 \\
\hline
\end{tabular}

shrinkage (Coleman and Flood, 1987; Terry et al., 1987). Other studies indicate that white matter loss as early as 60 years of age could be responsible for reduced brain volume (Guttmann et al., 1998; Salat et al., 1999, 2001; Jernigan et al., 2001). From the present study, it is unclear whether changes in gray or white matter or both account for decreased frontal lobe volume in older dogs. Additional studies are underway to examine white and gray matter aging in dogs as well as markers representing neuron, glial, or synapse loss in several brain regions.

Additional work also needs to be done to determine whether subregions of the frontal cortex atrophy at equal rates in the aging dog. Studies of age-related changes in subdivisions of the human prefrontal cortex in young, old, and Alzheimer's subjects indicate that the dorsolateral, inferior prefrontal, and orbitofrontal cortices are more vulnerable to age compared with other frontal subregions such as the precentral gyrus, frontal pole, and anterior cingulate gyrus (Raz et al., 1993, 1997; Xu et al., 2000; Salat et al., 2001; Tisserand et al., 2002).

Additional measures of nonfrontal brain regions are also needed to compare rates of regional cortical atrophy across the dog brain. The aging human brain is characterized by shrinkage of the hippocampal complex (Jack et al., 1992; Golomb et al., 1996; Petersen et al., 2000; Wolf et al., 2001), temporal lobes (Coffey et al., 1992; Jack et al., 1998; Black et al., 2000), corpus callosum (Weis et al., 1993; Hampel et al., 1998; Black et al., 2000; Yamauchi et al., 2000; Teipel et al., 2002), and cerebellar vermis (Raz et al., 1992, 1998a). In the present study, the occipital lobe showed no change in volume with age past maturity, indicating that age-related cortical atrophy occurs only in vulnerable brain regions including the frontal lobes and the hippocampus.

\section{Additional brain-behavior measures in aging dogs}

MRI studies of human brain aging indicate correlations between region-specific changes in brain volume and cognitive aging (Golomb et al., 1996; Fox et al., 1999; Petersen et al., 2000; Wolf et al., 2001). For example, impaired executive function is concomitant with decreased prefrontal volume in elderly subjects (Raz et al., 1998b; Head et al., 2002; Gunning-Dixon and Raz, 2003), but

\section{References}

executive functions also vary with volumetric changes in nonfrontal brain regions (Lombardi et al., 1999; Stuss and Alexander, 2000). Tests developed to assess executive functions in aging dogs suggested that inhibitory control (Tapp et al., 2003a), maintenance and manipulation of complex information in working memory (Tapp et al., 2003b), and rule induction and concept learning (Tapp et al., 2004), decrease with age. In the present study, decreased FLV was associated with impaired inhibitory control, maintenance and manipulation of complex working memory information, and, to a lesser degree, concept learning and rule induction. These functions are most likely not exclusive to the frontal lobes but involve cortical circuits that include other brain regions, such as the hippocampus. A similar relationship was observed between the cognitive measures and hippocampal volume, suggesting that the agerelated impairment in executive functions in the beagle could be attributable to decreased FLV, HCV, or both. Frontal lobe lesion studies are currently in progress to delineate the role of frontal lobes in executive functions in dogs.

The frontal lobes of the dog appear to be particularly sensitive to the aging process based on the neurological, neuroanatomical, and cognitive evidence presented. Additional work is required to clarify brain-behavior relationships in the canine model of aging, but these findings strongly suggest that the frontal lobes of the dog play a similar role to that observed in humans and are sensitive to aging.

Birrell JM, Brown VJ (2000) Medial frontal cortex mediates perceptual attentional set shifting in the rat. J Neurosci 20:4320-4324.

Black SE, Moffat SD, Yu DC, Parker J, Stanchev P, Bronskill M (2000) Callosal atrophy correlates with temporal lobe volume and mental status in Alzheimer's disease. Can J Neurol Sci 27:204-209.

Braak H, Braak E (1997) Staging of Alzheimer-related cortical destruction. Int Psychogeriatr 9 [Suppl 1]:257-261.

Bussière T, Friend PD, Sadeghi N, Wicinski B, Lin GI, Bouras C, Giannakopoulos P, Robakis NK, Morrison JH, Perl DP, Hof PR (2002) Stereological assessment of the total cortical volume occupied by amyloid deposits and its relationship with cognitive status in aging and Alzheimer's disease. Neuroscience 112:75-91.

Coffey CE, Wilkinson WE, Parashos IA, Soady SA, Sullivan RJ, Patterson LJ, Figiel GS, Webb MC, Spritzer CE, Djang WT (1992) Quantitative cerebral anatomy of the aging human brain: a cross-sectional study using magnetic resonance imaging. Neurology 42:527-536.

Coleman PD, Flood DG (1987) Neuron numbers and dendritic extent in normal aging and Alzheimer's disease. Neurobiol Aging 8:521-545.

Condon B, Grant R, Hadley D, Lawrence A (1988) Brain and intracranial cavity volumes: in vivo determination by MRI. Acta Neurol Scand 78:387-393.

Cummings BJ, Cotman CW (1995) Image analysis of $\beta$-amyloid "load" in Alzheimer's disease and relation to dementia severity. Lancet 346:1524-1528.

Cummings BJ, Head E, Afagh AJ, Milgram NW, Cotman CW (1996) Betaamyloid accumulation correlates with cognitive dysfunction in the aged canine. Neurobiol Learn Mem 66:11-23.

Delatour B, Gisquet-Verrier P (2001) Involvement of the dorsal anterior cingulate cortex in temporal behavioral sequencing: subregional analysis of the medial prefrontal cortex in rat. Behav Brain Res 126:105-114.

Dias R, Robbins TW, Roberts AC (1996) Primate analogue of the Wisconsin Card Sorting Test: effects of excitotoxic lesions of the prefrontal cortex in the marmoset. Behav Neurosci 110:872-886.

Fox MW (1971) Integrative development of brain and behavior in the dog, pp 135-148. Chicago: University of Chicago. 
Fox NC, Scahill R, Crum WR, Rossor MN (1999) Correlations between rates of brain atrophy and cognitive decline in AD. Neurology 52:1687-1689.

Ge Y, Grossman RI, Babb JS, Rabin ML, Mannon LJ, Kolson DL (2002) Age-related total gray matter and white matter changes in normal adult brain. Part I: volumetric MR imaging analysis. Am J Neuroradiol 23:1327-1333.

Golomb J, Kluger A, de Leon MJ, Ferris SH, Mittelman M, Cohen J, George AE (1996) Hippocampal formation size predicts declining memory performance in normal aging. Neurology 47:810-813.

González-Soriano J, García MP, Contreras-Rodríguez J, Martínez-Sainz P, Rodríguez-Veiga E (2001) Age-related changes in the ventricular system of the dog brain. Ann Anat 183:283-291.

Gunning-Dixon FM, Raz N (2003) Neuroanatomical correlates of selected executive functions in middle-aged and older adults: a prospective MRI study. Neuropsychologia 41:1929-1941.

Gur RC, Mozley PD, Resnick SM, Gottlieb GL, Kohn M, Zimmerman R, Herman G, Atlas S, Grossman R, Berretta D, Erwin R, Gur RE (1991) Gender differences in age effect on brain atrophy measured by magnetic resonance imaging. Proc Natl Acad Sci USA 88:2845-2849.

Guttmann CR, Jolesz FA, Kikinis R, Killiany RJ, Moss MB, Sandor T, Albert MS (1998) White matter changes with normal aging. Neurology 50:972-978.

Hampel H, Teipel SJ, Alexander GE, Horwitz B, Teichberg D, Schapiro MB, Rapoport SI (1998) Corpus callosum atrophy is a possible indicator of regions-specific and cell type-specific neuronal degeneration in Alzheimer disease: a magnetic resonance imaging analysis. Arch Neurol 55:193-198.

Head D, Raz N, Gunning-Dixon F, Williamson A, Acker JD (2002) Agerelated differences in the course of cognitive skill acquisition: the role of regional cortical shrinkage and cognitive resources. Psychol Aging 17:72-84.

Head E, Callahan H, Muggenburg BA, Cotman CW, Milgram NW (1998) Visual-discrimination learning ability and beta-amyloid accumulation in the dog. Neurobiol Aging 19:415-425.

Head E, McCleary R, Hahn FF, Milgram NW, Cotman CW (2000) Regionspecific age at onset of beta-amyloid in dogs. Neurobiol Aging 21:89-96.

Heilbroner PL, Kemper T (1990) Cytoarchitectonic distribution of senile plaques in three aged monkeys. Acta Neuropathol (Berl) 81:60-65.

Hou Y, White RG, Bobik M, Marks JS, Russell MJ (1997) Distribution of beta-amyloid in the canine brain. NeuroReport 8:1009-1012.

Iversen SD, Mishkin M (1970) Perseverative interference in monkeys following selective lesions of the inferior prefrontal convexity. Exp Brain Res 11:376-386.

Jack C, Peterson R, O’Brien P, Tangalos E (1992) MR-based hippocampal volumetry in the diagnosis of Alzheimer's disease. Neurology 42:183-188.

Jack CR, Peterson RC, Xu YC, O’Brien PC, Smith GE, Ivnik RJ, Tangalos EG, Kokmen E (1998) Rate of medial temporal lobe atrophy in typical aging and Alzheimer's disease. Neurology 51:993-999.

Jernigan TL, Archibald SL, Berhow MT, Sowell ER, Foster DS, Hesselink JR (1991) Cerebral structure on MRI, part 1: localization of age-related changes. Biol Psychiatry 29:55-67.

Jernigan TL, Archibald SL, Fennema-Notestine C, Gamst AC, Stout JC, Bonner J, Hesselink JR (2001) Effects of age on tissues and regions of the cerebrum and cerebellum. Neurobiol Aging 22:581-594.

Kracun I, Rosner H, Drnovsek V, Heffer-Lauc M, Cosovic C, Lauc G (1991) Human brain gangliosides in development, aging, and disease. Int J Dev Biol 35:289-295.

Kracun I, Kalanj S, Talan-Hranilovic J, Cosovic C (1992) Cortical distribution of gangliosides in Alzheimer's disease. Neurochem Int 20:433-438.

Kreiner J (1966) Reconstruction of neocortical lesions within the dog's brain: instructions. Acta Biol Exp (Warsz) 26:221-243.

Lombardi WJ, Anderson PJ, Sirocco KY, Rio DE, Gross RE, Umhau JC, Hommer DW (1999) Wisconsin Card Sorting Test performance following head injury: dorsolateral fronto-striatal circuit activity predicts perseveration. J Clin Exp Neuropsychol 21:2-16.

Milgram NW, Head E, Weiner E, Thomas E (1994) Cognitive functions and aging in the dog: acquisition of nonspatial visual tasks. Behav Neurosci 108:57-68.

Milner B (1982) Some cognitive effects of frontal lobe lesions in man. Philos Trans R Soc Lond B Biol Sci 286:211-226.

Murphy DG, DeCarli C, McIntosh AR, Daly E, Mentis MJ, Pietrini P,
Szczepanik J, Schapiro MB, Grady CL, Horwitz B, Rapoport SI (1996) Sex differences in human brain morphometry and metabolism: an in vivo quantitative magnetic resonance imaging and positron emission tomography study on the effect of aging. Arch Gen Psychiatry 53:585-594.

Petersen RC, Jack RC, Xu YC, Waring SC, O’Brien PC, Smith GE, Ivnik RJ Tangalos EG, Boeve BF, Kokmen E (2000) Memory and MRI-based hippocampal volumes in aging and AD. Neurology 54:581-587.

Petrides M (1991) Monitoring of selections of visual stimuli and the primate frontal cortex. Proc R Soc Lond B Biol Sci 246:293-298.

Petrides M (1995) Impairments on nonspatial self ordered and externally ordered working memory tasks after lesions of the mid-dorsal part of the lateral frontal cortex in the monkey. J Neurosci 15:359-375.

Pike CJ, Burdick D, Walencewicz AJ, Glabe CG, Cotman CW (1993) Neurodegeneration induced by $\beta$-amyloid peptides in vitro: the role of peptide assembly state. J Neurosci 13:1676-1687.

Raz N (1996) Neuroanatomy of aging brain: evidence from structural MRI. In: Neuroimaging II: clinical applications (Bigler E, ed), pp 153-174. New York: Plenum.

Raz N, Torres IJ, Spencer WD, White K, Acker JD (1992) Age-related regional differences in cerebellar vermis observed in vivo. Arch Neurol 49:412-416.

Raz N, Torres IJ, Spencer WD, Acker JD (1993) Pathoclysis in aging human cerebral cortex: evidence from in vivo MRI morphometry. Psychobiology (Austin, Tex) 21:151-160.

Raz N, Gunning FM, Head D, Dupuis JH, McQuain J, Briggs SD, Loken WJ, Thornton AE, Acker JD (1997) Selective aging of the human cerebral cortex observed in vivo: differential vulnerability of the prefrontal gray matter. Cereb Cortex 7:268-282.

Raz N, Dupuis JH, Briggs SD, McGavran C, Acker JD (1998a) Differential effects of age and sex on the cerebellar hemispheres and the vermis: a prospective MR study. Am J Neuroradiol 19:65-71.

Raz N, Gunning-Dixon FM, Head D, Dupuis JH, Acker JD (1998b) Neuroanatomical correlates of cognitive aging: evidence from structural magnetic resonance imaging. Neuropsychology 12:95-114.

Resnick SM, Goldszal AF, Davatzikos C, Golski S, Kraut MA, Metter EJ, Bryan $\mathrm{RN}$, Zonderman AB (2000) One-year age changes in MRI brain volumes in older adults. Cereb Cortex 10:464-472.

Salat DH, Kaye JA, Janowsky JS (1999) Prefrontal white and gray matter volumes in healthy aging and Alzheimer's disease. Arch Neurol 56:338-344.

Salat DH, Kaye JA, Janowsky JS (2001) Selective perseveration and degeneration within the prefrontal cortex in aging and Alzheimer disease. Arch Neurol 58:1403-1408.

Satou T, Cummings BJ, Head E, Nielson KA, Hahn FF, Milgram NW, Velazquez P, Cribbs DH, Tenner AJ, Cotman CW (1997) The progression of beta-amyloid deposition in the frontal cortex of the aged canine. Brain Res 774:35-43.

Struble RG, Price Jr DL, Cork LA, Price DL (1985) Senile plaques in cortex of aged normal monkeys. Brain Res 361:267-275.

Stuss DT, Alexander MP (2000) Executive functions and the frontal lobes: a conceptual view. Psychol Res 63:289-298.

Stuss DT, Floden D, Alexander MP, Levine B, Katz D (2001) Stroop performance in focal lesion patients: dissociation of processes and frontal lobe lesion location. Neuropsychologia 39:771-786.

Su M-Y, Head E, Brooks WM, Wang Z, Muggenburg BA, Adam GE, Sutherland R, Cotman CW, Nalcioglu O (1998) Magnetic resonance imaging of anatomic characteristics in a canine model of human aging. Neurobiol Aging 19:479-485.

Sullivan EV, Pfefferbaum A, Adalsteinsson E, Swan GE, Carmelli D (2002) Differential rates of regional brain change in callosal and ventricular size a 4-year longitudinal MRI study of elderly men. Cereb Cortex 12:438-445.

Talairach J, Tournoux P (1988) Co-planar stereotaxic atlas of the human brain. Stuttgart, Germany: Thieme.

Tapp PD, Siwak CT, Estrada J, Head E, Muggenburg BA, Cotman CW, Milgram NW (2003a) Size and reversal learning in the beagle dog as a measure of executive function and inhibitory control in aging. Learn Mem 10:63-73.

Tapp PD, Siwak CT, Estrada J, Holowachuk D, Milgram NW (2003b) Effects of age on measures of complex working memory span in the beagle dog (Canis familiaris) using two versions of a spatial list learning paradigm. Learn Mem 10:148-160. 
Tapp PD, Siwak CT, Head E, Cotman CW, Murphey H, Muggenburg BA, Ikeda-Douglas C, Milgram NW (2004) Concept abstraction in the aging dog: development of a protocol using a successive discrimination and size concept task. Behav Brain Res 153:199-210.

Teipel SJ, Bayer W, Alexander GE, Zebuhr Y, Teichberg D, Kulic L, Schapiro MB, Moller HJ, Rapoport SI, Hampel H (2002) Progression of corpus callosum atrophy in Alzheimer disease. Arch Neurol 59:243-248.

Terry RD, DeTeresa R, Hansen LA (1987) Neocortical cell counts in normal adult aging. Ann Neurol 21:530-539.

Tisserand DJ, Pruessner JC, Sanz Arigita EJ, van Boxtel MPJ, Evans AC, Jolles J, Uylings HB (2002) Regional frontal cortical volumes decrease differentially in aging: an MRI study to compare volumetric approaches and voxel-based morphometry. NeuroImage 17:657-669.

Vite CH, Insko EK, Schotland HM, Panckeri K, Hendricks JC (1997) Quantification of cerebral ventricular volume in English bulldogs. Vet Radiol Ultrasound 38:437-443.

Weis S, Kimbacher M, Wenger E, Neuhold A (1993) Morphometric analysis of the corpus callosum using MR: correlation of measurements with aging in healthy individuals. Am J Neuroradiol 14:637-645.
Winocur G, Moscovitch M (1990) Hippocampal and prefrontal cortex contributions to learning and memory: analysis of lesion and aging effects on maze learning in rats. Behav Neurosci 104:544-551.

Wolf H, Grunwald M, Kruggel F, Riedel-Heller SG, Angerhofer S, Hojjatoleslami A, Hensel A, Arendt T, Gertz H (2001) Hippocampal volume discriminates between normal cognition: questionable and mild dementia in the elderly. Neurobiol Aging 22:177-186.

Xu J, Kobayashi S, Yamaguchi S, Iijima K, Okada K, Yamashita K (2000) Gender effects on age-related changes in brain structure. Am J Neuroradiol 21:112-118.

Yamauchi H, Fukuyama H, Nagahama Y, Katsumi Y, Hayashi T, Oyanagi C, Konishi J, Shio H (2000) Comparison of the pattern of atrophy of the corpus callosum in frontotemporal dementia, progressive supranuclear palsy, and Alzheimer's disease. J Neurol Neurosurg Psychiatry 69:623-629.

Yoshino T, Uchida K, Tateyama S, Yamaguchi R, Nakayama H, Goto N (1996) A retrospective study of canine senile plaques and cerebral amyloid angiopathy. Vet Pathol 33:230-234. 